\title{
Reduced neural gating of respiratory sensations is associated with increased dyspnoea perception
}

\author{
To the Editor:
}

According to the neural gating model of respiratory sensations, breathing occurs under normal conditions automatically without reaching consciousness (gating-out). This neural filter mechanism prevents the brain from being flooded with irrelevant respiratory sensations leaving sufficient neural processing capacities for everyday activities [1,2]. However, in some circumstances, breathing can become conscious either voluntarily (e.g. attention, meditation) or due to increased respiratory demand (e.g. exacerbations, respiratory disease). In such cases, respiratory information is no longer filtered out but transmitted to higher brain centres which leads to the allocation of attentional resources towards the breathing sensation and brings it to conscious awareness (gating-in) [1,2]. Similar to the neural gating in other modalities, such as auditory/visual/somatosensory gating $[3,4]$, this respiratory gating mechanism is, therefore, the neural basis for monitoring respiratory functioning and a pre-requisite for subsequent adaptive behaviour, such as medication intake or physician visits. Anxiety, which is prevalent in patients with dyspnoea $[1,5]$, has been shown to be associated with reduced neural gating of respiratory sensations, suggesting gating deficits to be a potential mechanism for the documented over-perception of dyspnoea in anxious individuals $[6,7]$. The neural gating model of respiratory sensations implies that decreased neural gating of respiratory sensations is associated with increasing dyspnoea [4]. However, this implication has never been tested and was investigated in the present study by using respiratory-related evoked potentials (RREP) in the electroencephalogram (EEG) while additionally exploring potential effects of anxiety.

22 healthy participants (15 females; mean \pm SD age $19.59 \pm 2.42$ years) were tested after providing written consent (ethical approval: G-2015-12-409). Trait anxiety was measured using the State-Trait Anxiety Inventory [8] with higher scores indicating higher anxiety levels. Participants, with a noseclip attached, respired via a mouthpiece through a breathing circuit. The circuit consisted of a two-way non-rebreathing valve with the inspiratory port connected via tubing to a pneumotachograph, a loading manifold (all Hans Rudolph Inc., Shawnee, KS, USA) and occlusion device (Aspire Products, Gainesville, FL, USA)[6]. The occlusion device was used to trigger paired occlusions within a single inspiration. Breathing frequency, inspiratory time $\left(\mathrm{T}_{\mathrm{I}}\right)$, inspiratory mouth pressure $\left(P_{\mathrm{I}}\right)$ and airflow $\left(V^{\prime}\right)$ were recorded continuously. All participants underwent two experimental blocks, each consisting of a no dyspnoea condition (baseline) followed by three counterbalanced conditions resulting in very mild dyspnoea $\left(5 \mathrm{cmH} \mathrm{H}_{2} \mathrm{O} \cdot \mathrm{L}^{-1} \cdot \mathrm{s}^{-1}\right)$, mild dyspnoea $\left(10 \mathrm{cmH}_{2} \mathrm{O} \cdot \mathrm{L}^{-1} \cdot \mathrm{s}^{-1}\right)$ and moderate dyspnoea $\left(15 \mathrm{cmH}_{2} \mathrm{O} \cdot \mathrm{L}^{-1} \cdot \mathrm{s}^{-1}\right)$ (figure 1a). These were elicited by adding inspiratory resistive loads to the loading manifold. The 129-channel EEG (Philips EGI, Eugene, OR, USA) was continuously measured (sampling rate $250 \mathrm{~Hz}$; reference $\mathrm{Cz}$ ). After each condition, ratings of dyspnoea intensity and unpleasantness were obtained via a visual analogue scale ranging from 0 ("not noticeable/not unpleasant") to 100 ("maximally imaginable intensity/unpleasantness") [9].

The neural gating of respiratory sensations was assessed in all conditions by eliciting RREPs in the EEG by applying paired inspiratory occlusions (duration $150 \mathrm{~ms}$; inter-stimulus interval $500 \mathrm{~ms}$ ) randomly to every second to fifth inspiration [4]. Evoked RREP components with short latencies $(<130 \mathrm{~ms})$ represent the arrival and initial processing of afferent respiratory sensory information to the somatosensory cortex while RREP components with longer latencies $(>130 \mathrm{~ms})$ are thought to reflect the subsequent higher-order cortical processing of that afferent information [4]. Neural gating is evidenced by a reduced RREP N1

@ERSpublications

Reduced respiratory neural gating is associated with increased dyspnoea, particularly in high anxious individuals http://ow.ly/xW4B30k34KR

Cite this article as: Herzog M, Sucec J, Van Diest I, et al. Reduced neural gating of respiratory sensations is associated with increased dyspnoea perception. Eur Respir J 2018; 52: 1800559 [https://doi.org/10.1183/ 13993003.00559-2018]. 
a)

Study design

Ratings followed by 2-min break

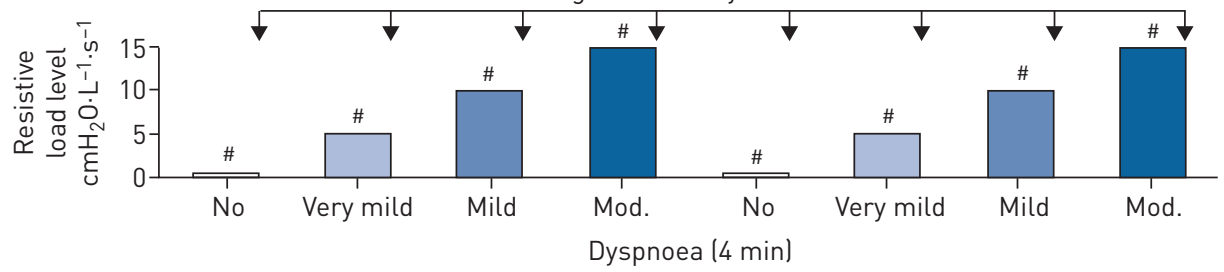

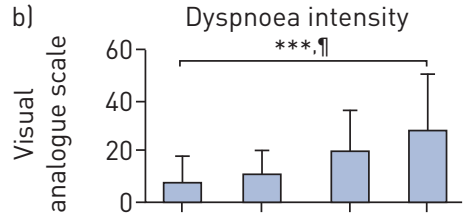

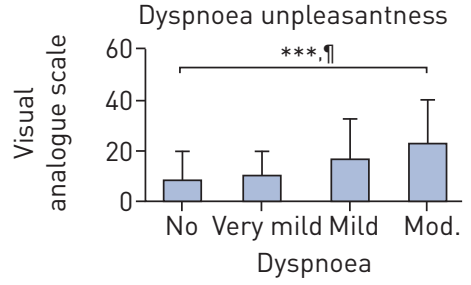

d)
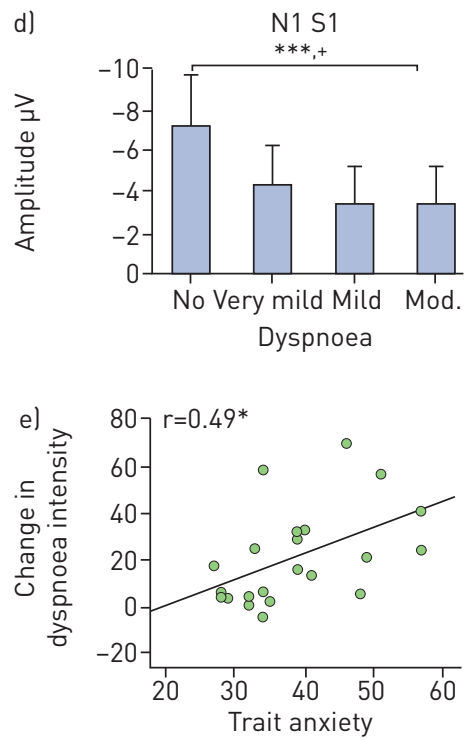
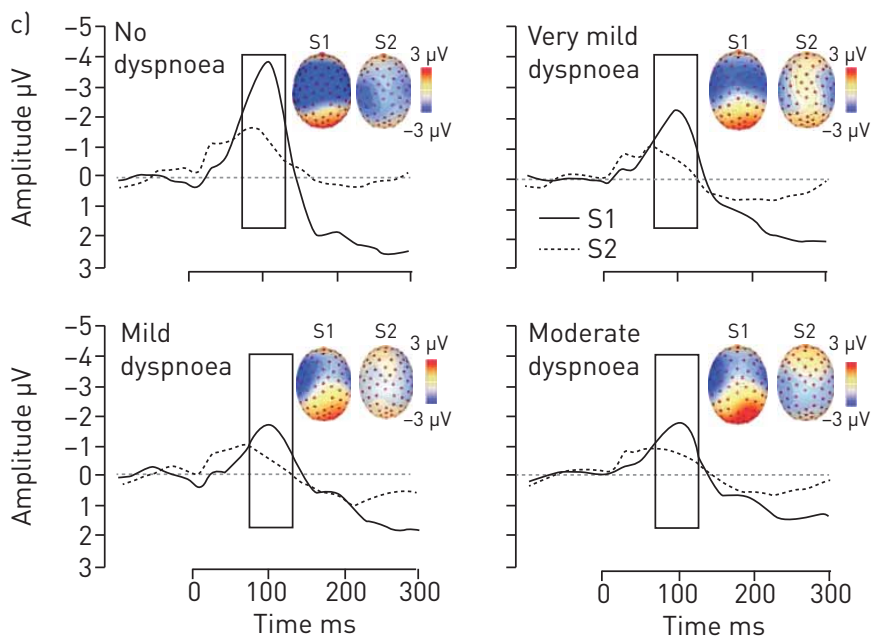

N1 S2
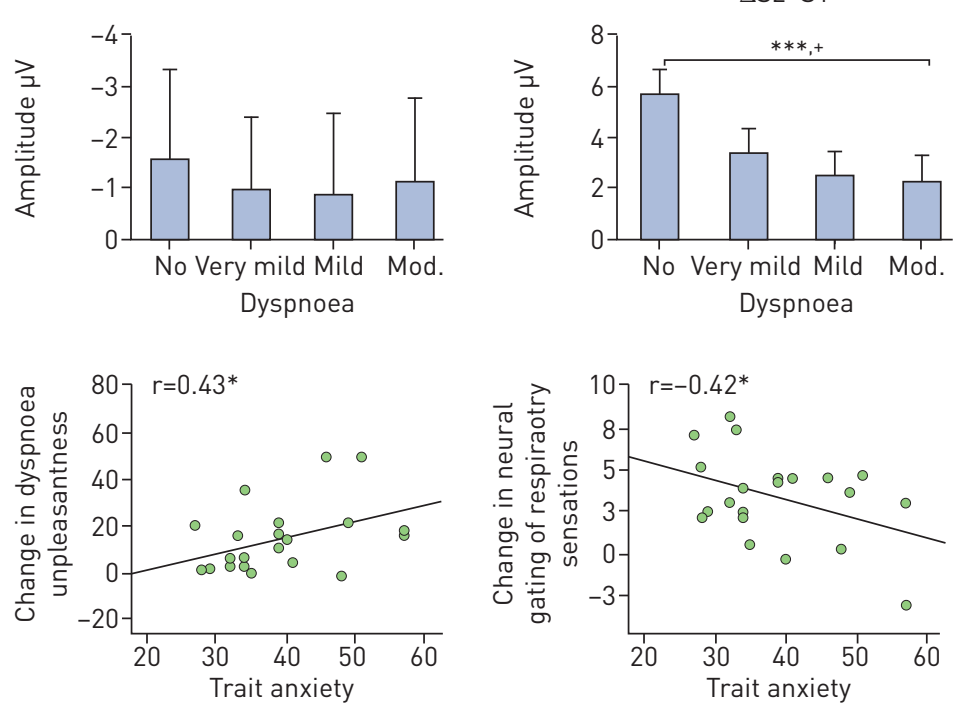

FIGURE 1 a) Schematic presentation of the study design for one exemplary participant. \#: measurements of respiratory-related evoked potentials (RREPs) elicited by paired occlusions (on average 28.76 paired occlusions were analysed per condition). The conditions of very mild, mild and moderate dyspnoea were presented in counterbalanced order across participants. b) Mean \pm SD ratings of dyspnoea intensity and unpleasantness for conditions of no dyspnoea (baseline), very mild dyspnoea $\left(5 \mathrm{cmH}_{2} \mathrm{O} \cdot \mathrm{L}^{-1} \cdot \mathrm{s}^{-1}\right)$, mild dyspnoea $\left(10 \mathrm{cmH}_{2} \mathrm{O} \cdot \mathrm{L}^{-1} \cdot \mathrm{s}^{-1}\right)$ and moderate dyspnoea $\left(15 \mathrm{cmH}_{2} \mathrm{O} \cdot \mathrm{L}^{-1} \cdot \mathrm{s}^{-1}\right.$ ). c) Group mean respiratory-related evoked potential $(\mu \mathrm{V})$ for the first (S1) and second occlusion (S2) for conditions of no dyspnoea, very mild dyspnoea, mild dyspnoea and moderate dyspnoea. RREPs were obtained by applying filters (high-pass $0.1 \mathrm{~Hz}$, low-pass $30 \mathrm{~Hz}$, notch $50 \mathrm{~Hz}$ ), artefact corrections and an average-re-referencing procedure to the data. This was followed by the extraction (200 ms pre- to $1000 \mathrm{~ms}$ post-occlusion onset) and averaging of the occlusion-related electroencephalogram epochs for S1 and S2 separately. d) Averaged N1 peak amplitudes $(\mu \mathrm{V})$ for the first occlusion (S1), second occlusion (S2) and neural gating of respiratory sensations (N1 difference scores S2-S1) for conditions of no dyspnoea, very mild dyspnoea, mild dyspnoea and moderate dyspnoea. e) Correlations between trait anxiety and changes in ratings of dyspnoea intensity, dyspnoea unpleasantness and neural gating of respiratory sensations from baseline to the moderate dyspnoea condition. Trait anxiety was measured with the validated Dutch version of the State-Trait Anxiety Inventory. Changes in dyspnoea intensity and unpleasantness were calculated by subtracting the ratings of the no dyspnoea condition from the moderate dyspnoea condition. Changes in neural gating were calculated by subtracting the N1 difference scores (S2-S1) of the moderate dyspnoea condition from the N1 difference scores (S2-S1) of the no dyspnoea condition. Higher values indicate a stronger increase of dyspnoea intensity, dyspnoea unpleasantness and neural gating from baseline to the moderate dyspnoea condition. The correlation demonstrates that increased trait anxiety is related to increased ratings of dyspnoea intensity and unpleasantness as well as to decreased neural gating. ${ }^{*}: p \leqslant 0.05 ;{ }^{* * *}: p<0.001$. ${ }^{\text {I: }}$ significant positive linear trend; ${ }^{+}$: significant negative linear trend. 
peak amplitude of the second occlusion (S2) compared to the first occlusion (S1) [4] with N1 amplitudes being partly related to attentional processes (figure 1c) [10].

EEG data were processed offline using BESA Research 6.0 (BESA GmbH, Gräfelfing, Germany) as previously described [6]. Considering previous reports [4, 7, 11], the RREP N1 peak amplitudes of S1 and S2 were identified in the centrolateral region at a latency around $80-135 \mathrm{~ms}$. As in previous studies in the respiratory [10] and other modalities $[12,13]$, neural gating was quantified as N1 amplitude difference score S2-S1, with higher difference scores indicating stronger neural gating. After testing the assumptions for all statistical tests, repeated-measures analyses of variance with the within factor condition (no/very $\mathrm{mild} / \mathrm{mild} /$ moderate dyspnoea) were calculated in SPSS (IBM Corp., Armonk, NY, USA) to investigate differences in outcome variables. A priori polynomial contrasts were calculated to investigate trends. Finally, explorative correlations (Pearson's $r$ ) between anxiety and changes in ratings of dyspnoea and the $\mathrm{N} 1$ difference score S2-S1 from baseline to the moderate dyspnoea condition were tested. The level of significance was $\mathrm{p} \leqslant 0.05$.

A significant difference between conditions was observed for ratings of dyspnoea ( $\mathrm{p}$-values $<0.001$ ). Polynomial contrasts demonstrated a positive linear trend for both dyspnoea intensity and unpleasantness ( $\mathrm{p}$-values $<0.001$ ) suggesting increased dyspnoea with increasing load levels (figure $1 \mathrm{~b}$ ).

All respiratory variables showed a significant difference between conditions ( $p$-values $<0.001$ ). Polynomial contrasts showed a negative linear trend with increasing load levels for frequency in breaths per min (mean \pm SD; $14.33 \pm 4.08,13.64 \pm 4.14,13.09 \pm 3.77,12.81 \pm 3.61), P_{\mathrm{I}, \max }$ in $\mathrm{cmH}_{2} \mathrm{O}$ (mean $\pm \mathrm{SD} ;-1.29 \pm 0.43$, $-3.70 \pm 1.02,-5.42 \pm 1.50,-6.92 \pm 1.87)$ and $V^{\prime}$ in $\mathrm{L} \cdot \mathrm{s}^{-1}$ (mean $\pm \mathrm{SD} ; 0.41 \pm 0.12,0.35 \pm 0.12,0.32 \pm 0.10,0.28$ \pm 0.08 ) (p-values $<0.001$ ). A positive linear trend was found for $T_{\mathrm{I}}$ in $\mathrm{s}$ (mean $\pm \mathrm{SD} ; 1.85 \pm 0.62,2.21 \pm 0.85$, $2.40 \pm 0.77,2.58 \pm 0.96 ; \mathrm{p}<0.001)$ with increasing load levels. These respiratory changes converge with commonly observed respiration patterns during increasing load levels [14].

A significant difference between conditions was found for the N1 amplitude of $\mathrm{S} 1(\mathrm{p}<0.001)$ with no effect for S2 ( $p=0.19)$ (figure 1d). Polynomial contrasts revealed a negative linear trend for the N1 amplitude of S1 $(p<0.001)$ suggesting decreasing neural processing of the S1 occlusion when increasing load levels require increasing attentional and neural processing resources (gating-in) (figure 1d). Most importantly, this was paralleled by a significant difference between conditions in the N1 difference score S2-S1 $(\mathrm{p}<0.001)$. Polynomial contrasts showed a negative linear trend $(\mathrm{p}<0.001)$ demonstrating decreasing neural gating with increasing load levels (figure 1d).

Finally, significant correlations were observed between anxiety and changes in ratings of perceived dyspnoea intensity, unpleasantness and N1 difference scores S2-S1 (p-values $\leqslant 0.05$ ), demonstrating higher anxiety to be associated with increased dyspnoea ratings and reduced neural gating of respiratory sensations under increasing load levels (figure 1e).

The present findings suggest that reduced neural gating of respiratory sensations, as quantified by decreased N1 difference scores S2-S1, is associated with increasing levels of resistive load-induced dyspnoea. These findings converge with previous research on other neural gating modalities showing an association between reduced sensory gating and acute painful cold pressor stimulation [15]. Notably, higher anxiety was associated with stronger increases in dyspnoea reports, as well as stronger reductions in neural gating during increasing load levels, which confirms previous observations on either dyspnoea reports or neural gating alone [5-7].

The present study has several notable implications. Most importantly, the findings suggest that neural gating may be a potential neural mechanism contributing to increasing levels of dyspnoea. This provides experimental support for the neural gating model of respiratory sensations and might partly be related to attentional processes [1, 2]. Moreover, the findings support the view of a gating deficit as a potential mechanism for the common observation of over-perception of dyspnoea in more anxious individuals $[1,2,5]$. Subsequently, the present findings suggest a potential neural target for pharmacological and/or non-pharmacological treatments which necessitates further studies.

The present study was conducted in healthy participants, restricting the generalisability of the findings to dyspnoeic patient populations. Furthermore, resistive-loaded breathing elicited only one quality of dyspnoea ("work/effort to breathe") whereas other qualities involving different physiological pathways (i.e. "chest tightness" or "air hunger") were not evaluated [2]. Thus, additional qualities and magnitudes of dyspnoea should be examined in future studies (e.g. $\mathrm{CO}_{2}$ inhalation, exercise-induced dyspnoea).

In summary, this study is the first to demonstrate that reduced neural gating of respiratory sensations is associated with increasing load-elicited dyspnoea levels, particularly in high anxious individuals. 
Michaela Herzog ${ }^{1,4}$, Josef Sucec ${ }^{1,4}$, Ilse Van Diest ${ }^{1}$, Omer Van den Bergh ${ }^{1}$, Pei-Ying Sarah Chan ${ }^{2}$, Paul Davenport ${ }^{3}$ and Andreas von Leupoldt $\oplus^{1}$

${ }^{1}$ Health Psychology, University of Leuven, Leuven, Belgium. ${ }^{2}$ Dept of Occupational Therapy and Healthy Aging Centre, Chang Gung University, Taoyuan, Taiwan. ${ }^{3}$ Dept of Physiological Sciences, University of Florida, Gainesville, FL, USA. ${ }^{4}$ Both authors contributed equally to this work.

Correspondence: Andreas von Leupoldt, Research Group Health Psychology, University of Leuven, Tiensestraat 102, 3000 Leuven, Belgium. E-mail: andreas.vonleupoldt@kuleuven.be

Received: March 202018 | Accepted after revision: May 112018

Acknowledgement: We thank Thierry Troosters for his valuable and critical evaluation of the present findings and previous versions of this manuscript.

Author contributions: A. von Leupoldt, P. Davenport, O. Van den Bergh, I. Van Diest, J. Sucec and M. Herzog conceptualised the research design and research protocol. M. Herzog and J. Sucec were responsible for the data collection. M. Herzog, J. Sucec and A. von Leupoldt were responsible for the statistical analysis. M. Herzog, J. Sucec and A. von Leupoldt had access to all the data in the study and take responsibility for the integrity of the data as well as the data analysis. M. Herzog and J. Sucec created the figure. J. Sucec, M. Herzog and A. von Leupoldt wrote the manuscript. J. Sucec, M. Herzog, A. von Leupoldt, I. Van Diest, O. Van den Bergh, P-Y.S. Chan and P. Davenport provided feedback on the protocol, interpretation of the study results and on the manuscript. All authors read and approved the final version.

Conflict of interest: A. von Leupoldt reports grants from Research Fund KU Leuven, Belgium (research grants: STRT/13/ 002 and DBOF/14/021), Herculesstichting, Belgium (research infrastructure grant: AKUL/13/07), the Flemish Government, Belgium (long-term structural research funding Methusalem grant: METH/15/011), and Research Foundation - Flanders, Belgium (research grant: G0A3718N), during the conduct of the study.

Support statement: This study was supported by grants from the Research Fund KU Leuven, Belgium (STRT/13/002 and $\mathrm{DBOF} / 14 / 021)$, the Research Foundation - Flanders, Belgium $(\mathrm{G} 0 \mathrm{~A} 3718 \mathrm{~N})$, by an infrastructure grant from the Herculesstichting, Belgium (AKUL/13/07) and by the "Asthenes" long-term structural funding Methusalem grant (METH/15/011) by the Flemish Government, Belgium. The funders had no role in the study design, data collection, data analysis, data interpretation, or writing of the manuscript. M. Herzog, J. Sucec and A. von Leupoldt had full access to all data. All authors had the final responsibility for the decision to submit the manuscript for publication. Funding information for this article has been deposited with the Crossref Funder Registry.

\section{References}

1 Laviolette L, Laveneziana P. Dyspnoea: a multidimensional and multidisciplinary approach. Eur Respir J 2014; 43: $1750-1762$

2 O’Donnell DE, Banzett RB, Carrieri-Kohlman V, et al. Pathophysiology of dyspnea in chronic obstructive pulmonary disease: a roundtable. Proc Am Thorac Soc 2007; 4: 145-168.

3 Adler LE, Waldo MC, Freedman R. Neurophysiologic studies of sensory gating in schizophrenia: comparison of auditory and visual responses. Biol Psychiatry 1985; 20: 1284-1296.

4 Chan PYS, Davenport PW. Respiratory-related evoked potential measures of respiratory sensory gating. J Appl Physiol 2008; 105: 1106-1113.

5 von Leupoldt A. Treating anxious expectations can improve dyspnoea in patients with COPD. Eur Respir J 2017; 50: $1-4$.

6 Chan PYS, von Leupoldt A, Bradley MM, et al. The effect of anxiety on respiratory sensory gating measured by respiratory-related evoked potentials. Biol Psychol 2012; 91: 185-189.

7 Chan P-YS, Cheng C-H, Hsu S-C, et al. Respiratory sensory gating measured by respiratory-related evoked potentials in generalized anxiety disorder. Front Psychol 2015; 6: 1-6.

8 van der Ploeg HM. The development and validation of a Dutch inventory for the measurement of anxiety: “de Zelf-Beoordehngs Vragenhjst (STAI-DY)." Tijdschr Voor Psychiatr 1982; 24: 576-588.

9 Aitken RC. Measurement of feelings using visual analogue scales. Proc R Soc Med 1969; 62: 989-993.

10 Chenivesse C, Chan P-Y, Tsai H-W, et al. Negative emotional stimulation decreases respiratory sensory gating in healthy humans. Respir Physiol Neurobiol 2014; 204: 50-57.

11 Redolfi S, Raux M, Donzel-Raynaud C, et al. Effects of upper airway anaesthesia on respiratory-related evoked potentials in humans. Eur Respir J 2005; 26: 1097-1103.

12 Rentzsch J. Test-retest reliability of P50, N100 and P200 auditory sensory gating in healthy subjects. Int J Psychophysiol 2008; 67: 81-90.

13 Smith DA, Boutros NN, Schwarzopf SB. Reliability of P50 auditory event-related potential indices of sensory gating. Psychophysiology 1994; 31: 495-502.

14 Peiffer C, Poline J-B, Thivard L, et al. Neural substrates for the perception of acutely induced dyspnea. Am J Respir Crit Care Med 2001; 163: 951-957.

15 Johnson MR, Adler LE. Transient impairment in P50 auditory sensory gating induced by a cold-pressor test. Biol Psychiatry 1993; 33: 380-387. 